\title{
Empirical Relations Predicting Human and Rat Tissue:Air Partition Coefficients of Volatile Organic Compounds
}

\author{
Cécil J. W. Meulenberg and Henk P. M. Vijverberg ${ }^{1}$ \\ Research Institute of Toxicology, Utrecht University, P.O. Box 80.176, NL-3508 TD Utrecht, The Netherlands
}

Received September 20, 1999; accepted March 5, 2000

\begin{abstract}
E mpirical Relations Predicting H uman and R at T issue:A ir Partition Coefficients of Volatile Organic Compounds. Meulenberg, C. J. W., and Vijverberg, H. P. M. (2000). Toxicol. Appl. Pharmacol. 165, 206-216.
\end{abstract}

Based on the hypothesis that tissue partitioning of volatile organic compounds (VOCs) is due to lipophilic and hydrophilic interactions with tissue components, empirical relations are established between olive oil $\left(\boldsymbol{P}_{\text {oil:air }}\right)$, saline $\left(\boldsymbol{P}_{\text {saline:air }}\right)$, and tissue partition coefficients $\left(\boldsymbol{P}_{\text {tissueair }}\right)$ for human and rat tissues. R eported values of partition coefficients of a wide range of VOC $s$ with distinct chemical structures $(n=137)$ have been compiled from the literature. Bilinear regression analysis shows that partition coefficients of VOC s in human blood, brain, fat, liver, kidney, and muscle tissues are well described by a linear combination of $\boldsymbol{P}_{\text {oil:air }}$ and $\boldsymbol{P}_{\text {salineair }}$ with tissue-specific regression coefficients. The regression coefficient associated with the hydrophilic component of VOC partitioning in rat tissues is systematically higher than that of human tissues. For the human model, tissue concentrations calculated from predicted partition coefficients are generally within a factor 4 of tissue concentrations calculated from experimentally observed partition coefficients. These results demonstrate that, without prior knowledge of tissue composition, it is possible to obtain estimates of human tissue partition coefficients of VOC $s$ with an accuracy that is in the same range as that commonly used in risk assessment. $\odot 2000$ Academic Press

Key Words: Organic solvents; tissue partition coefficients; human PBPK modeling.

Knowledge of the distribution of chemicals over different body compartments contributes to the understanding of the risk of toxic effects. Ambient exposure to volatile organic compounds (VOCs), particularly in the occupational setting, may cause adverse, neurotoxic effects (reviewed by Mikkelsen, 1997; White and Proctor, 1997). Despite their neurotoxic potential, only a few studies have addressed the relation between exposure and brain concentrations of VOCs, which constitute a large, heterogeneous class of chemicals. Alternatively, physiologically based pharmacokinetic (PBPK) models have been

${ }^{1}$ To whom correspondence should be addressed. Fax: 3130253 5077; E-mail: H.Vijverberg@RITOX.vet.uu.nl. used to describe the relation between inhalation exposure to and tissue concentrations of VOCs (Andersen, 1991; Krishnan and Andersen, 1994; Gargas et al., 1995). However, a specific brain compartment is often lacking in PBPK models. The detailed modeling of brain concentrations is hampered by a general lack of knowledge of brain tissue partition coefficients.

Partitioning between blood and a specific tissue depends on the relative affinities of a compound for blood and for the tissue. For volatile substances it is more convenient to determine tissue:air partition coefficients (Sato and Nakajima, 1979a), and blood:tissue partition coefficients are defined as the ratio of the blood:air partition coefficient $\left(P_{\text {bloodair }}\right)$ and the tissue:air partition coefficient $\left(P_{\text {tissue:arr }}\right)$. Since the relative proportions and the basic composition of tissue constituents vary among tissues, the prediction of tissue partitioning on a rational basis requires detailed knowledge of tissue composition (Poulin and Krishnan, 1995a,b). However, a simplified approach supposes that tissue partitioning of nonreactive chemicals is determined completely by lipophilic and hydrophilic interactions of compounds with tissue constituents. It has been shown before that such a simple approach successfully applies to a set of 12 volatile anesthetics. The $P_{\text {tissue:air }}$ of these volatile anesthetics in human tissues can be described as linear combinations of $P_{\text {saline:air }}$ and $P_{\text {olive oil:air }}$ (Droz, 1978).

Demonstration of the applicability of this approach to tissue partitioning of VOCs in general would provide a basis for predicting tissue partitioning without prior knowledge of the tissue composition. Despite the neurotoxic potential of VOCs, human tissue partition coefficients for industrial important organic solvents, e.g., the alkylbenzenes, appear to be lacking in the literature and, in general, brain tissue partition coefficients have been reported for a limited number of compounds only. Since brain concentrations may be a key issue for the risk assessment of VOCs, detailed knowledge on VOC partitioning in brain tissue is required.

Here we have compiled published values of partition coefficients for a large number of VOCs in olive oil and saline, as well as partition coefficients in rat and human blood, fat, brain, liver, muscle, and kidney tissues. Using linear regression analysis, empirical relations are established between the partitioning of VOCs in tissues, olive oil, and saline, based on the 
approach used for volatile anesthetics before (Droz, 1978). The results demonstrate that VOC partitioning into tissues can be described by a linear combination of the saline and oil partition coefficients with tissue- and species-dependent coefficients.

\section{METHODS}

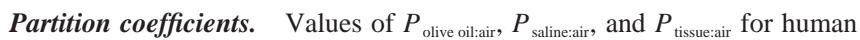
and rat blood, fat, brain, liver, muscle, and kidney were compiled from various sources. The reported values are compiled in Table 1. Values for $P_{\text {olive oil:air, }}$ $P_{\text {saline:air }}$, and human $P_{\text {bloodair }}$ for various organic solvents were first reported by Droz (1978) and by Sato and Nakajima (1979a,b). Steward et al. (1973) compiled human tissue partition coefficients for various anesthetics. Paterson and Mackay (1989) compiled water solubility values for some compounds. Extensive data sets containing partition coefficients for various human tissues and a large number of compounds were reported by Fiserova-Bergerova et al. (1984), Perbellini et al. (1985), and Fiserova-Bergerova and Diaz (1986). Some sources contained values of human $P_{\text {bloodair }}$ for a few compounds only. These values were included when the corresponding $P_{\text {oilair }}$ and $P_{\text {saline:air }}$ were reported as well (Johanson and Dynésius, 1988; Järnberg and Johanson, 1995; Nihlén and Johanson, 1995). A large set of rat tissue partition coefficients with corresponding values of $P_{\text {oil:air }}$ and $P_{\text {saline:air }}$ for various VOCs published by Gargas et al. (1989) was included and extended with rat tissue partition coefficients for ethylbenzene (Tardif et al., 1997). Kaneko et al. (1994) reported rat data for alcohols and esters, which were compiled and supplemented with additional values for ketones by Poulin and Krishnan (1996a,b). Pierce et al. (1996) reported partition coefficients of aromatic hydrocarbons in human and rat fat tissue. Values for human and rat tissue partition coefficients of volatile anesthetics and related compounds, e.g., fluorinated alkanes, are scattered over various references (Eger and Eger, 1985; Coburn and Eger, 1986; Fassoulaki and Eger, 1986; Lerman et al., 1986, 1987; Eger, 1987; Strum and Eger, 1987; Yasuda et al., 1989; Taheri et al., 1993; Chortkoff et al., 1994; Eger et al., 1994; Liu et al., 1994; Fang et al., 1996, 1997a,b). Two compounds with extremely low water, oil, and tissue partition coefficients, perfluoropropane and perfluoropentane (Eger et al., 1994), were not included in Table 1. In general, reported partition coefficients have been determined in vitro by headspace gas chromatography at $37^{\circ} \mathrm{C}$ in a vial equilibration technique (Sato and Nakajima, 1979a) or by a modification of this method (Gargas et al., 1989). In the compilation of saline partition coefficients it was noted that in many cases partitioning in saline and in water is considered to be identical. Although $P_{\text {saline:air }}$ may be slightly lower than $P_{\text {waterair }}$ (Steward et al., 1973; Lerman et al., 1983), they are considered equivalent here. When values of partition coefficients were available from multiple sources, the mean was calculated and used in this study.

Regression analysis. $\quad P_{\text {tissue:air }}$ is described as a bilinear function of $P_{\text {olive oil:air }}$ and $P_{\text {saline:air }}($ Droz, 1978) according to:

$$
P_{\text {tissue:air }}=\alpha_{\mathrm{o}} P_{\text {olive oil:air }}+\alpha_{\mathrm{s}} P_{\text {saline:air }}+c .
$$

The coefficients $\alpha_{\mathrm{o}}$ and $\alpha_{\mathrm{s}}$ in Eq. (1) represent the tissue-specific contributions of the lipophilic and hydrophilic interactions to the solubility of the compounds in tissue. The constant $c$ was included in the equation to avoid errors in the slope of the regression plane, which occurred in fitting the same equation with zero intercept. Although $c$ has no specific physical meaning it may be required to compensate for systematic errors in tissue, oil, or saline partition coefficients. Tissue partitioning according to Eq. (1) was fitted using weighted bilinear regression; i.e., each tissue partition coefficient value was divided by its own value to ensure equal weights of individual compounds in the regressions. Estimated values of the regression coefficients $\alpha_{\mathrm{o}}$ and $\alpha_{\mathrm{s}}$ and of the constant $c$ are reported with their coefficients of variation $(\mathrm{CV}, \%)$, and regressions are presented with their correlation coefficients $\left(R^{2}\right)$. Cross-correlation between fitted parameters was also monitored to judge the quality of the regressions and possible redundancy of parameters. To analyze the appli- cability of the estimated regression coefficients for the prediction of tissue partition coefficients, the ratio of predicted and observed values was plotted logarithmically against $\log P_{\text {oilair }}$ and against $\log P_{\text {saline:air }}$, and the mean value and 2.5 and 97.5 percentiles were determined for each tissue. All regression analyses were performed using SigmaPlot 3.02 software (Jandel Scientific Software, SPSS Inc., Chicago, IL).

\section{RESULTS}

\section{Oil and Saline Partition Coefficients}

All values of $P_{\text {oil:air }}$ and $P_{\text {saline:air }}$ of VOCs, obtained from the literature and compiled in Table $1(n=137)$, are graphically presented in Fig. 1. From the clustering of points near the origin in Fig. 1 it is clear that many of the VOCs have relatively small $P_{\text {oil:air }}$ and $P_{\text {saline:air }}$ values. Compounds which are highly lipophilic or highly hydrophilic always have small corresponding $P_{\text {saline:air }}$ or $P_{\text {oil:air }}$, respectively. Only a few compounds, e.g., 2-butoxyethanol (Fig. 1, compound 112), combine intermediate lipophilicity and hydrophilicity. Due to the inverse relation between oil and saline partition coefficients, points are found in a region of the plane limited by a hyperbolic curve. Although water-soluble VOCs are underrepresented in the total set of data, the scatter of the data (see Fig. 1, inset) indicates that the correlation between oil and saline partition coefficients is small for the data compiled and that $P_{\text {oil:air }}$ and $P_{\text {saline:air }}$ are largely independent descriptors of VOC properties.

\section{Human Tissue Partition Coefficients}

Experimental values of partition coefficients of VOCs, available from the literature, in human blood $(n=109)$ and human fat, liver, brain, muscle, and kidney tissues $(n=28-41$; see Table 1) were used in regression analysis. For each of the human tissues the relation between $P_{\text {tissue:air }}$ and the corresponding $P_{\text {oil:air }}$ and $P_{\text {saline:air }}$ was evaluated by bilinear regression according to Eq. (1). The regressions for $P_{\text {fat:air }}, P_{\text {bran:air }}$, and $P_{\text {blood:air }}$ are plotted in three-dimensional graphs in Fig. 2. For fat tissue and blood the slopes of the regression planes are mainly determined by $\alpha_{\mathrm{o}}$ and $\alpha_{\mathrm{s}}$, respectively. Although the slope of the regression plane of brain tissue partition coefficients is intermediate between those of blood and fat tissue partition coefficients, $\alpha_{\mathrm{s}}$ appears to be the main regression coefficient for brain tissue partitioning. Predicted $P_{\text {tissue:air }}$ values for human muscle and kidney are within planes with an intermediate orientation similar to that for brain (not shown). The results of the regressions for human tissue partition coefficients (Table 2) show that, except for fat, tissue partitioning is mainly predicted by the $P_{\text {saline:air }}$, according to large values of $\alpha_{\mathrm{s}}$ over small values of $\alpha_{\mathrm{o}}$. For all tissues, except liver, the regressions yielded good correlation coefficients $\left(R^{2}=0.92-0.99\right)$, and the crosscorrelation between the estimated values of the parameters $\alpha_{\mathrm{s}}$ and $\alpha_{\mathrm{o}}$ was very small $(<0.04)$. For human liver, only $\alpha_{\mathrm{o}}$ has been determined. Partition coefficients available for human liver were strongly biased toward more lipophilic compounds (see Table 1 and Fig. 3). Therefore, a reliable estimate of $\alpha_{\mathrm{s}}$ 


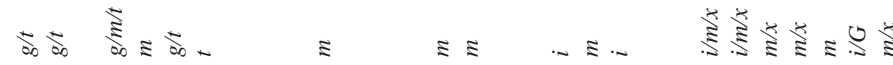

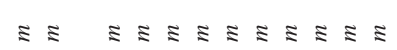
这

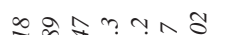

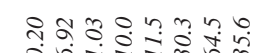

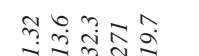

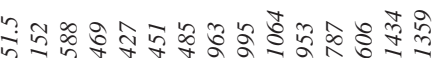

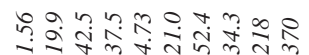
ڤै:

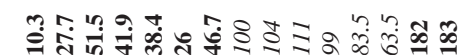

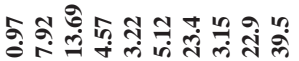

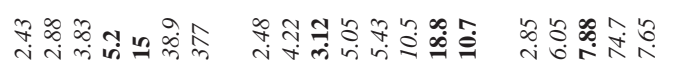

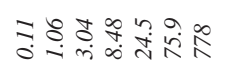

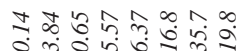
sin

ニ ம்:

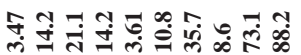
$+a n=0$

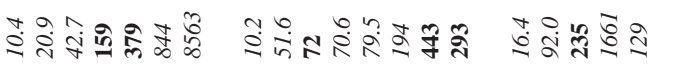

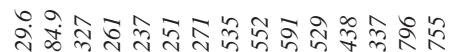

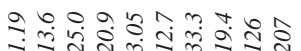

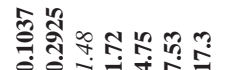
กู่

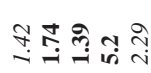

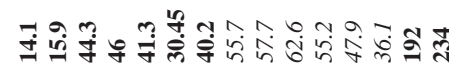

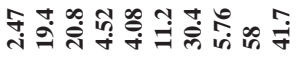
$33 \stackrel{3}{z}-$ 03333

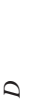

\section{$\stackrel{5}{2} \infty$}

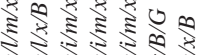

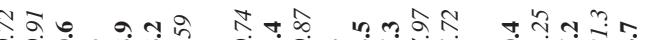

บํำำ

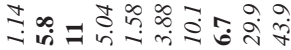

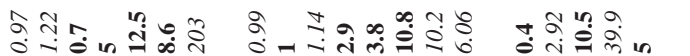

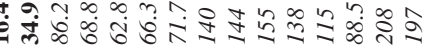

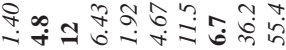

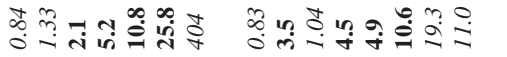

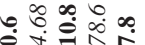

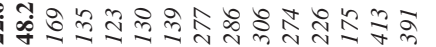

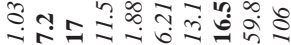

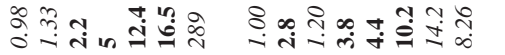

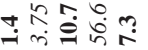

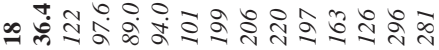

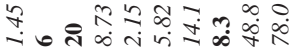

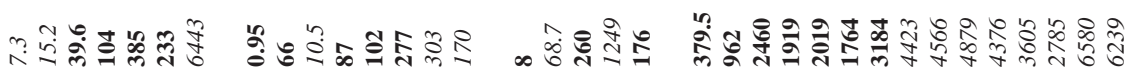

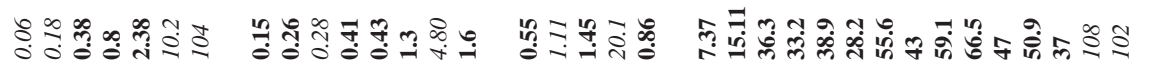

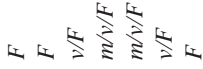

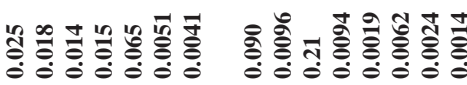
a... กิำ (⿻)

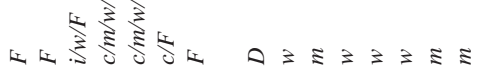
ล. ปิ
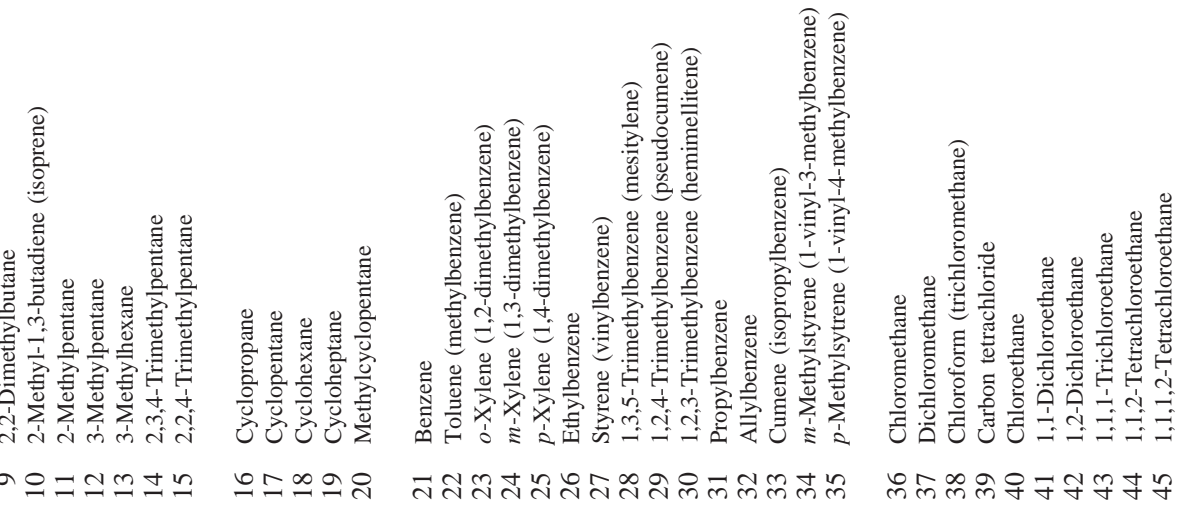

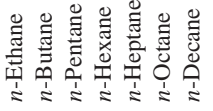 - Nm+nor}


$\Xi ミ ミ ミ ミ ミ ミ ミ ミ ミ ミ ミ ミ$

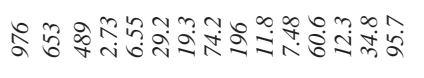

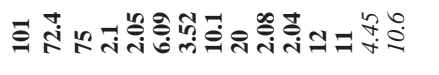

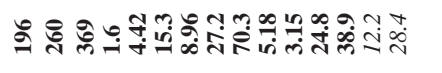

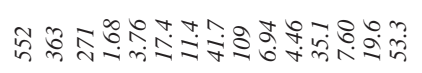

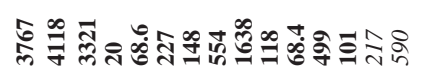

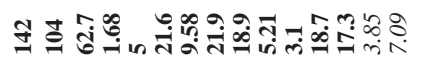

$\Xi ミ ミ ミ ミ \quad \geqq ミ \quad \geqq$

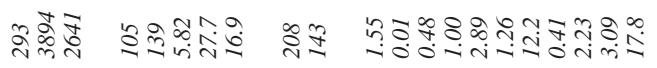

m웟

守尔

केषें

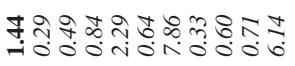

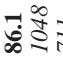

는

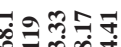

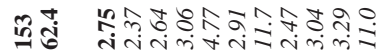

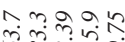

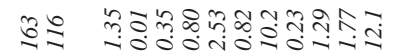

촗ํำ

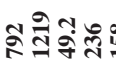

เุ่ง

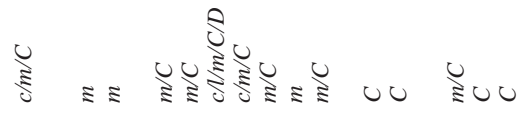

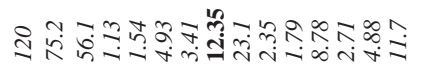

$\dot{i} \neq \overline{2}$

$ミ ミ ミ$

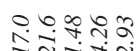

กิะ

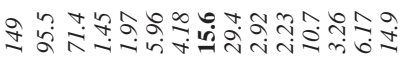

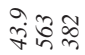

बें

它文

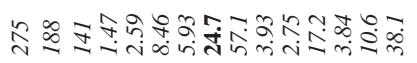

₹

ㄴำ

$\sum_{\infty}^{\infty}$

(nt)

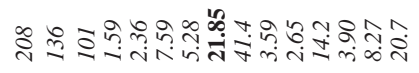

$\stackrel{i}{\infty} \infty$

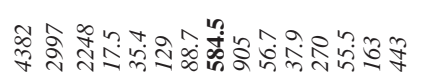

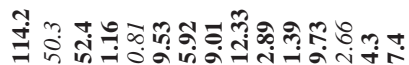

$\hat{n} \cong$

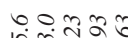

in-

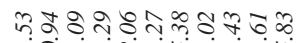

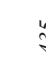

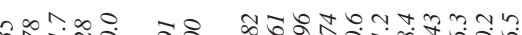

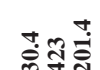

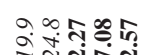

a

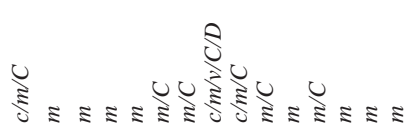

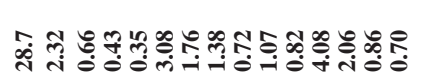

$\geqq \cup$

$\Xi ミ ミ ミ ミ$

क

Su

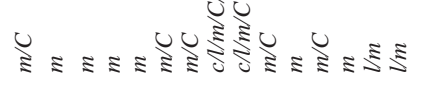

te $e^{2}$

in

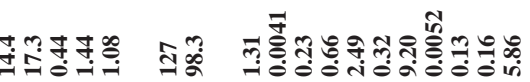

ミしu

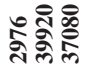
ㅇำ ชัย

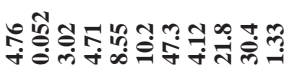
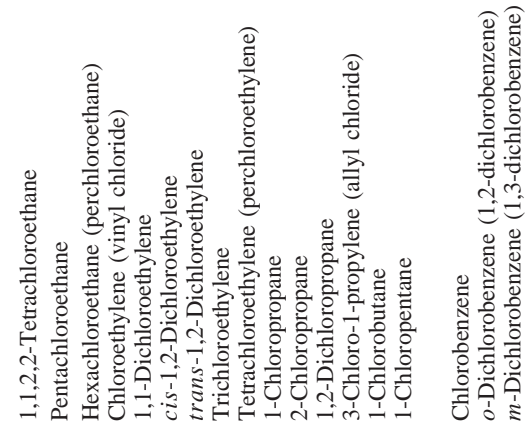

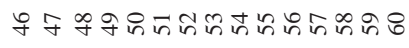
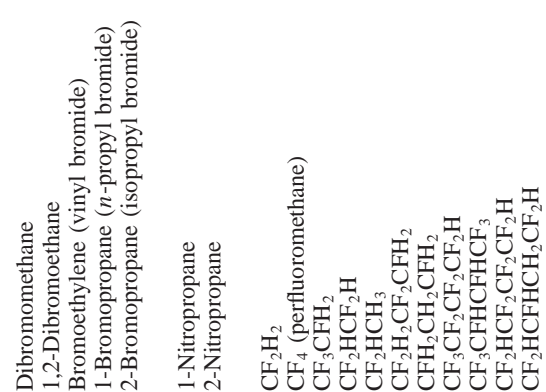

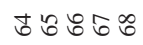

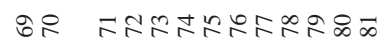

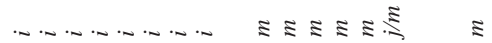

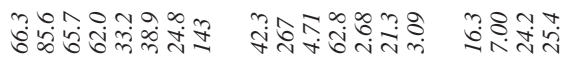

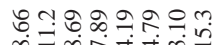

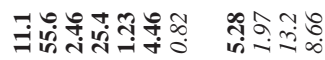

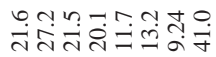

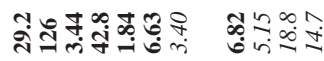

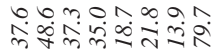

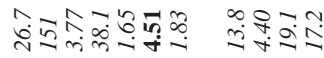

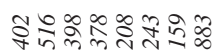

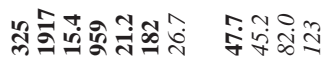

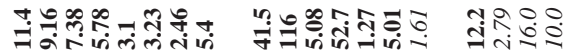

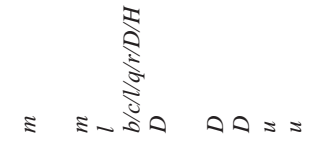

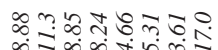

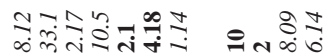

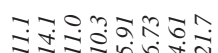

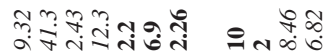

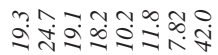

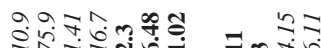

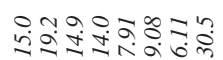

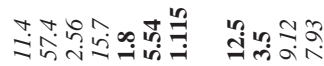

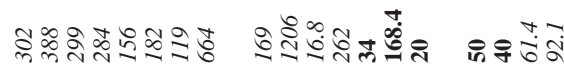

๒สำำกำำ

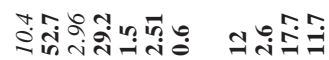

$\sum_{1}^{2}$

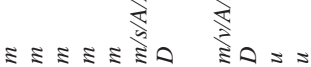

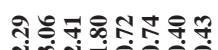

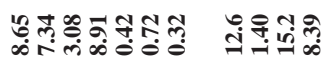

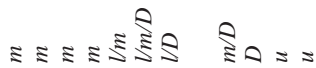

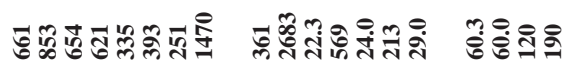
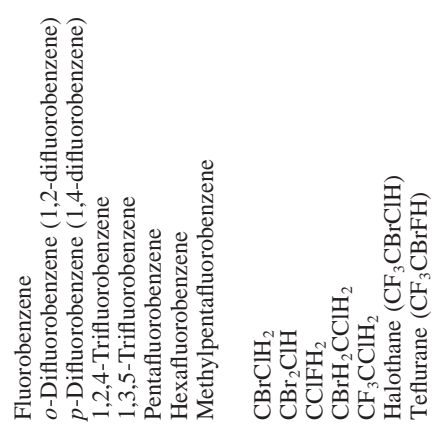

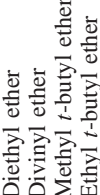

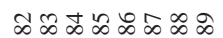




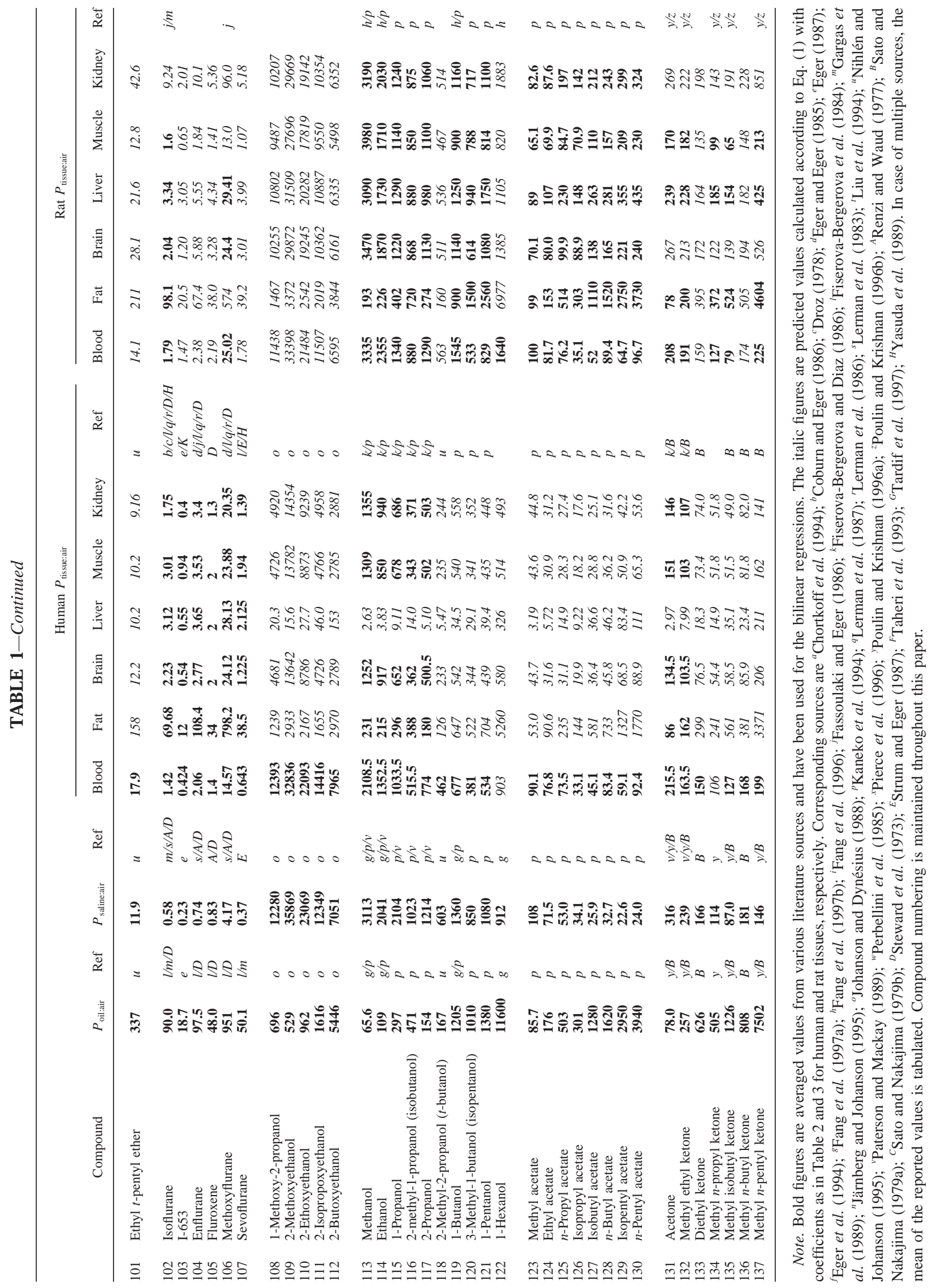




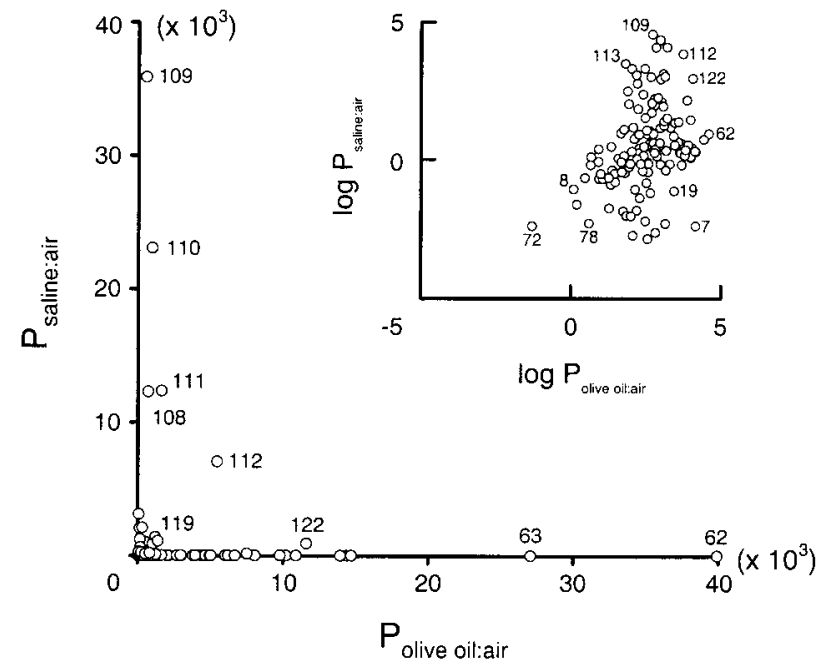

FIG. 1. Scatter plot of $P_{\text {oil:air }}$ vs $P_{\text {saline:air }}$ for all compounds presented in Table $1(n=137)$. The numbered points refer to specific compounds in Table 1 . The inset shows the scatter of data points in a log-log presentation.

could not be obtained and a linear regression was performed on $P_{\text {liver:air }}$ and $P_{\text {oil:air }}$ values to obtain estimates of $\alpha_{\mathrm{o}}$ and $c$ for human liver $\left(R^{2}=0.88\right)$. With the exception of fat tissue, the estimated values of the intercept $c$ were all smaller than 1 . The intercepts showed large coefficients of variation and a large cross-correlation with the other regression parameters $(\leq 0.22)$, illustrative of the minor contribution of this parameter to tissue partitioning.

The equations fitted to tissue partitioning of VOCs have been internally validated from plots of the logarithm of the ratio of predicted and observed values. Figure 3 is a double logarithmic plot against $P_{\text {oil:air }}$ and against $P_{\text {saline:air }}$. For all tissues, the data points are scattered around zero and their distributions do not deviate from normal $(p>0.10)$. The means of the logarithmic ratios do not differ from 0 statistically, with the exception of blood and fat $(p=0.03$ and 0.02 , respectively). These deviations are caused by a few outliers, e.g., ethylene for fat (Fig. 3, compound 8). In order to assess the reliability of the predicted values, the 2.5 and 97.5 percentiles of the ratios of predicted and reported partition coefficients were calculated for each tissue. The resulting 95\% confidence range is indicated in each panel of Fig. 3. Tissue concentrations were calculated from the quotient of predicted and from the quotient of experimental $P_{\text {tissue:air }}$ and $P_{\text {blood:air }}$. The tissue concentrations predicted by the model are within a factor of 4.0 from the tissue concentrations calculated from experimental data for $95 \%$ of the compounds for human brain, muscle, kidney, and fat tissue. The results indicate that the partitioning of VOCs in human tissues can be calculated on the basis of saline and olive oil partitioning according to Eq. (1) with a good predictive power.

Equation (1) was also applied to data on the partitioning of four terpenes (Falk et al., 1990) and four gases (Steward et al., 1973) in human blood. Both chemical classes are not included in the data of Table 1 . The ratios of predicted versus experimental $P_{\text {blood:air }}$ were 1.4 for $\alpha$-pinene, 1.4 for $\beta$-pinene, 1.1 for 3 -carene, and 1.0 for limonene. For the gases, the ratios of predicted versus experimental $P_{\text {bloodair }}$ were 1.3 for $\mathrm{Kr}, 0.9$ for $\mathrm{Xe}, 2.9$ for nitrogen, and 1.0 for nitrous oxide. These results provide support for a more general applicability of Eq. (1) to blood partitioning of volatile organic compounds. Partition coefficients of the gases in other human tissues are very small (Steward et al., 1973) and are in the same order of magnitude as the fitted intercept $c$ (Table 2). This results in overestimation of the human tissue partition coefficients for gases.
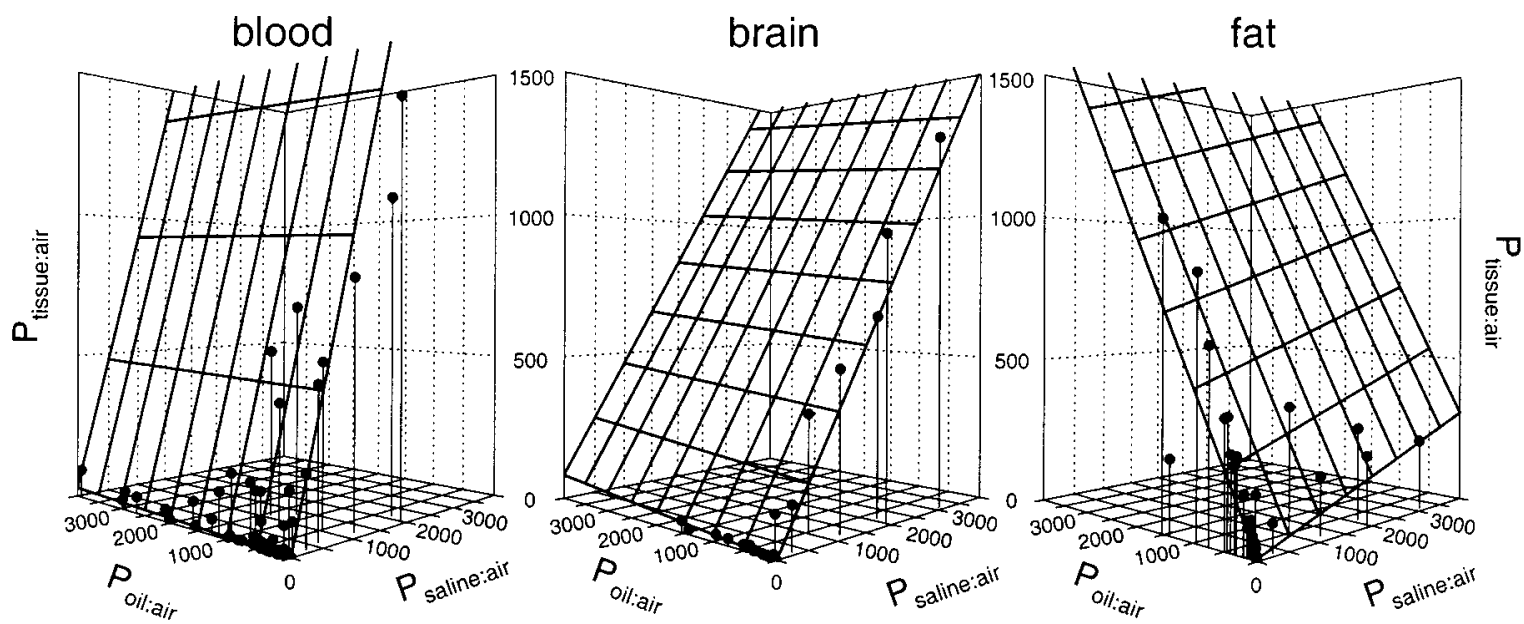

FIG. 2. Three-dimensional representation of the bilinear regression between $P_{\text {oil:air }}, P_{\text {saline:air }}$, and reported values of human $P_{\text {bloodair }}, P_{\text {brain:air }}$, and $P_{\text {fatair }}$ according to Eq. (1). The grids represent the fitted planes of predicted partition coefficients and the dots represent experimental values. Identically spaced grids are given in the horizontal and vertical planes. Note that the orientation of the plane fitted for brain tissue partitioning is intermediate between that fitted for blood and fat tissue partitioning. Vertical lines are drawn from the horizontal plane to indicate the position of the data points. 
TABLE 2

Results of Bilinear Regressions for the Partitioning of VOC s into Human Tissues Fitted According to Eq. (1)

\begin{tabular}{|c|c|c|c|c|c|c|c|c|}
\hline Tissue & $n$ & $\alpha_{\mathrm{o}}$ & $\mathrm{CV}(\%)$ & $\alpha_{\mathrm{s}}$ & $\mathrm{CV}(\%)$ & $c$ & $\mathrm{CV}(\%)$ & $R^{2}$ \\
\hline Blood & 109 & 0.0072 & 18 & 0.898 & 2 & 0.03 & 2094 & 0.99 \\
\hline Fat & 41 & 0.447 & 6 & 0.075 & 33 & 6.59 & 88 & 0.92 \\
\hline Brain & 35 & 0.020 & 16 & 0.380 & 3 & 0.94 & 69 & 0.98 \\
\hline Liver & 28 & 0.028 & 8 & nd & nd & 0.79 & 47 & 0.88 \\
\hline Muscle & 35 & 0.014 & 20 & 0.384 & 3 & 0.94 & 64 & 0.99 \\
\hline Kidney & 34 & 0.011 & 22 & 0.400 & 3 & 0.69 & 77 & 0.98 \\
\hline
\end{tabular}

Note. The number of compounds used in the regression $(n)$, the regression coefficients $\alpha_{\mathrm{o}}$ and $\alpha_{\mathrm{s}}$, and the constant $c$ together with their coefficients of variation $(\mathrm{CV})$ are tabulated. The correlation coefficients of the regressions $\left(R^{2}\right)$ are also indicated. nd, not determined.

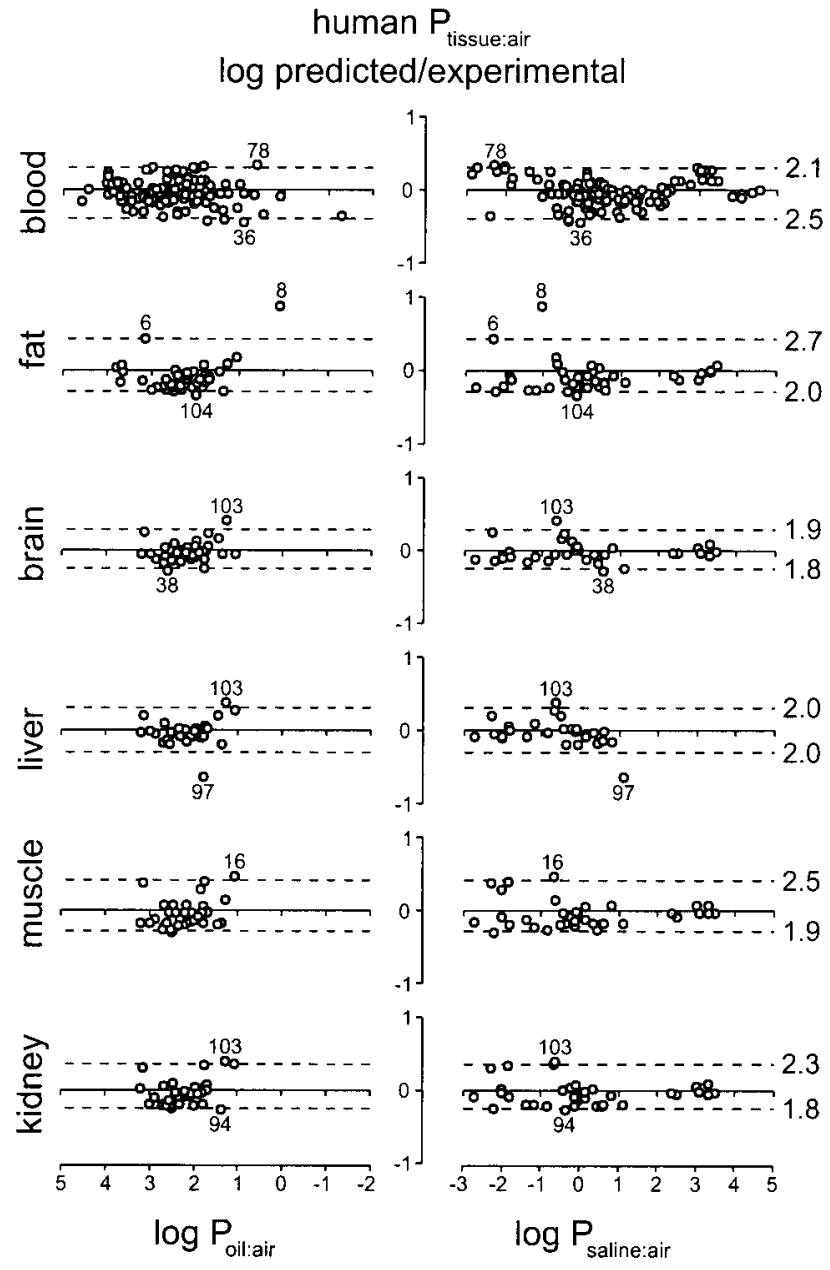

FIG. 3. Double-log representation of the ratio of predicted and experimentally determined $P_{\text {tissue:air }}$ against $P_{\text {oil:air }}$ and against $P_{\text {saline:air }}$ for human blood, fat, brain, liver, muscle, and kidney as indicated. Results are presented in two dimensions for a clear insight into the distribution of all points relative to the fitted regression planes. The 2.5 and 97.5 percentiles are drawn (dashed lines) and the values of the percentiles are indicated at the right for each tissue. Outliers are identified by their compound numbers.

\section{Rat Tissue Partition Coefficients}

For rat blood, fat, liver, and muscle, large numbers $(n=$ 76-92) of partition coefficients of VOCs are available from the literature (Table 1). However, data for rat brain $(n=19)$ and kidney $(n=16)$ appear to be less abundant. The regression planes obtained by fitting Eq. (1) to the data for $P_{\text {fat:air }}$, $P_{\text {brain:air, }}$, and $P_{\text {blood:air }}$ are plotted in three-dimensional graphs in Fig. 4. Qualitatively, the results are similar to those obtained for human tissue partition coefficients (see Fig. 2). The slopes of the planes describing fat tissue and blood partitioning are determined mainly by $\alpha_{\mathrm{o}}$ and $\alpha_{\mathrm{s}}$, respectively. For brain and other tissues intermediate slopes with $\alpha_{\mathrm{s}}$ as the main regression coefficient were obtained. The results of the regressions are summarized in Table 3. The quality of the regressions for rat tissues was not as good as that for human tissues, as indicated by slightly lower correlation coefficients $\left(R^{2}=0.82-0.93\right)$. The cross-correlation between the fitted parameters $\alpha_{\mathrm{o}}$ and $\alpha_{\mathrm{s}}$ was $<0.03$. The estimated values of the intercept were generally small, with the exception of the value for rat fat, which was estimated to be 9.4 and the coefficients of cross-correlation between the values estimated for the intercept and for the two other regression parameters ranged from 0.06 to 0.11 . For rat kidney and brain, the intercept could not be determined reliably, because of the lack of data on VOCs with small $P_{\text {oilair }}$ and $P_{\text {saline:air }}$ values (see Table 1 and Fig. 5). Despite the qualitative resemblance of the regressions of rat and human data, Table 3 shows a marked quantitative difference in the estimated values of $\alpha_{\mathrm{s}}$. Estimates of $\alpha_{\mathrm{s}}$ for rat brain, liver, muscle, and kidney are all in a narrow range and are approximately twofold the corresponding values of $\alpha_{\mathrm{s}}$ obtained for human tissues (see Table 2). Double logarithmic plots of the ratio of predicted and observed values show that the data points are scattered around zero for all tissues (Fig. 5), and their distributions do not deviate from normal $(p>0.10)$, except for rat fat $(p=$ 0.03 ). The means of the logarithmic ratios do not differ from 0 statistically with the exception of blood $(p<0.0001)$, fat $(p=0.01)$, and muscle $(p=0.02)$. These deviations are caused by outliers, e.g., $n$-ethane, $n$-butane, and $n$-decane for blood (compounds 1,2, and 7, respectively). Outliers for fat and muscle tissues are 1-nitropropane, 2-nitropropane (com- 


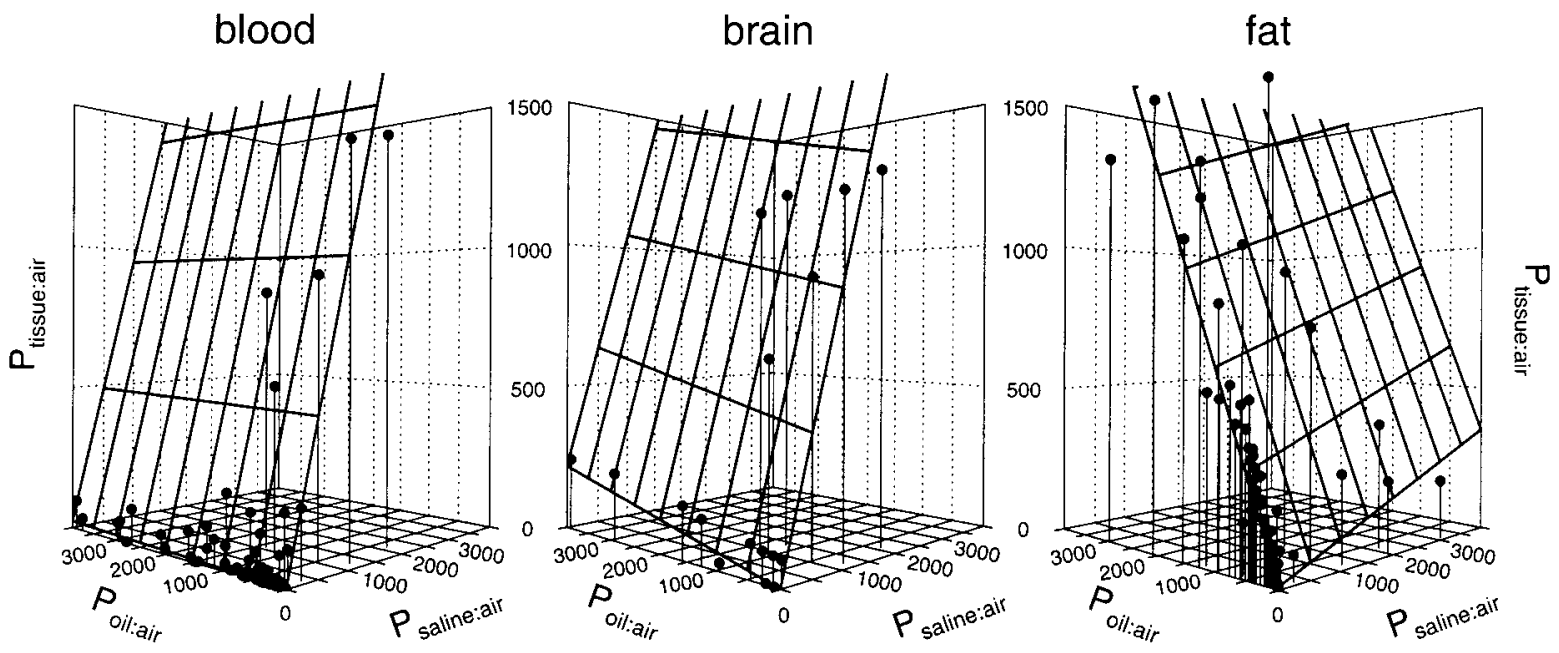

FIG . 4. Three-dimensional representation of the bilinear regression between $P_{\text {oil:air }}, P_{\text {saline:air, }}$ and reported values of rat $P_{\text {blood:air }}, P_{\text {brain:air }}$, and $P_{\text {fat:air }}$ according to Eq. (1). The grids represent the fitted planes of predicted partition coefficients and the dots represent experimental values. Identically spaced grids are given in the horizontal and vertical planes. The orientation of the plane fitted for brain tissue partitioning is intermediate between that fitted for blood and fat tissue partitioning. Note that the regression of rat $P_{\text {brain:air }}$ depends more steeply on $P_{\text {saline:air }}$ than the regression of human $P_{\text {brain:air }}$ (see Fig. 2). Vertical lines are drawn from the horizontal plane to indicate the position of the data points.

pounds 69 and 70), and several esters (compounds 127-130). The 2.5 and 97.5 percentiles of the ratios of predicted and reported partition coefficients were calculated. The resulting 95\% confidence range is indicated in each panel of Fig. 5. Predicted concentrations in liver, muscle, kidney, and fat tissue were within a factor of 5.0 from concentrations calculated from experimental values for $95 \%$ of the compounds.

\section{DISCUSSION}

The present results show that it is possible to predict tissue partition coefficients of VOCs from a simple linear combination of olive oil and saline partitioning, which is very similar to the method used before to predict partitioning of anesthetics in human tissues (Droz, 1978). Regression coefficients are estimated from data on a large set of VOCs, selected only by availability in the literature and not by the chemical nature of the compounds. The good quality of the regressions and the reliability of the predictions made by the model, particularly for human tissues, show that this approach is applicable to volatile compounds in general. In addition, the evaluation of VOC partitioning in six different human tissues and in the homologous rat tissues allows for making comparisons between tissues and between the two species.

Other studies describing empirical relations between tissue, oil, and saline partition coefficients, e.g., for chlorinated alkanes in human blood (Sato and Nakajima, 1979b) and for VOCs in several rat tissues (Gargas et al., 1989), have performed regressions on logarithmically transformed data using equations for tissue partitioning similar to Eq. (1). However, a major problem with the use of logarithmic equations of the type $\log P_{\text {tissue:air }}=\mathrm{a} \log P_{\text {oil:air }}+\mathrm{b} \log P_{\text {saline:air }}$ is that the tissue partition coefficient is implicitly assumed to be proportional to the product of water and oil partition coefficients (i.e., $P_{\text {tissue:air }}$ $\propto P_{\text {oil:air }} * P_{\text {saline:air }}$, or $P_{\text {tissue:saline }} \propto P_{\text {oil:air }}$ ). The meaning of such

TABLE 3

Results of Bilinear Regressions for the Partitioning of VOCs into Rat Tissues Fitted According to Eq. (1)

\begin{tabular}{|c|c|c|c|c|c|c|c|c|}
\hline Tissue & $n$ & $\alpha_{\mathrm{o}}$ & $\mathrm{CV}(\%)$ & $\alpha_{\mathrm{s}}$ & $\mathrm{CV}(\%)$ & $c$ & $\mathrm{CV}(\%)$ & $R^{2}$ \\
\hline Blood & 92 & 0.0054 & 19 & 0.931 & 4 & 1.16 & 87 & 0.93 \\
\hline Fat & 76 & 0.594 & 4 & 0.085 & 46 & 9.40 & 116 & 0.86 \\
\hline Brain & 19 & 0.054 & 27 & 0.832 & 6 & nd & nd & 0.90 \\
\hline Liver & 77 & 0.026 & 11 & 0.878 & 5 & 2.36 & 96 & 0.92 \\
\hline Muscle & 76 & 0.010 & 17 & 0.772 & 5 & 0.29 & 532 & 0.82 \\
\hline Kidney & 16 & 0.097 & 21 & 0.826 & 6 & nd & nd & 0.91 \\
\hline
\end{tabular}

Note. The number of compounds used in the regression $(n)$, the regression coefficients $\alpha_{\mathrm{o}}$ and $\alpha_{\mathrm{s}}$, and the constant $c$ together with their coefficients of variation $(\mathrm{CV})$ are tabulated. The correlation coefficients of the regressions $\left(R^{2}\right)$ are also indicated. nd, not determined. 


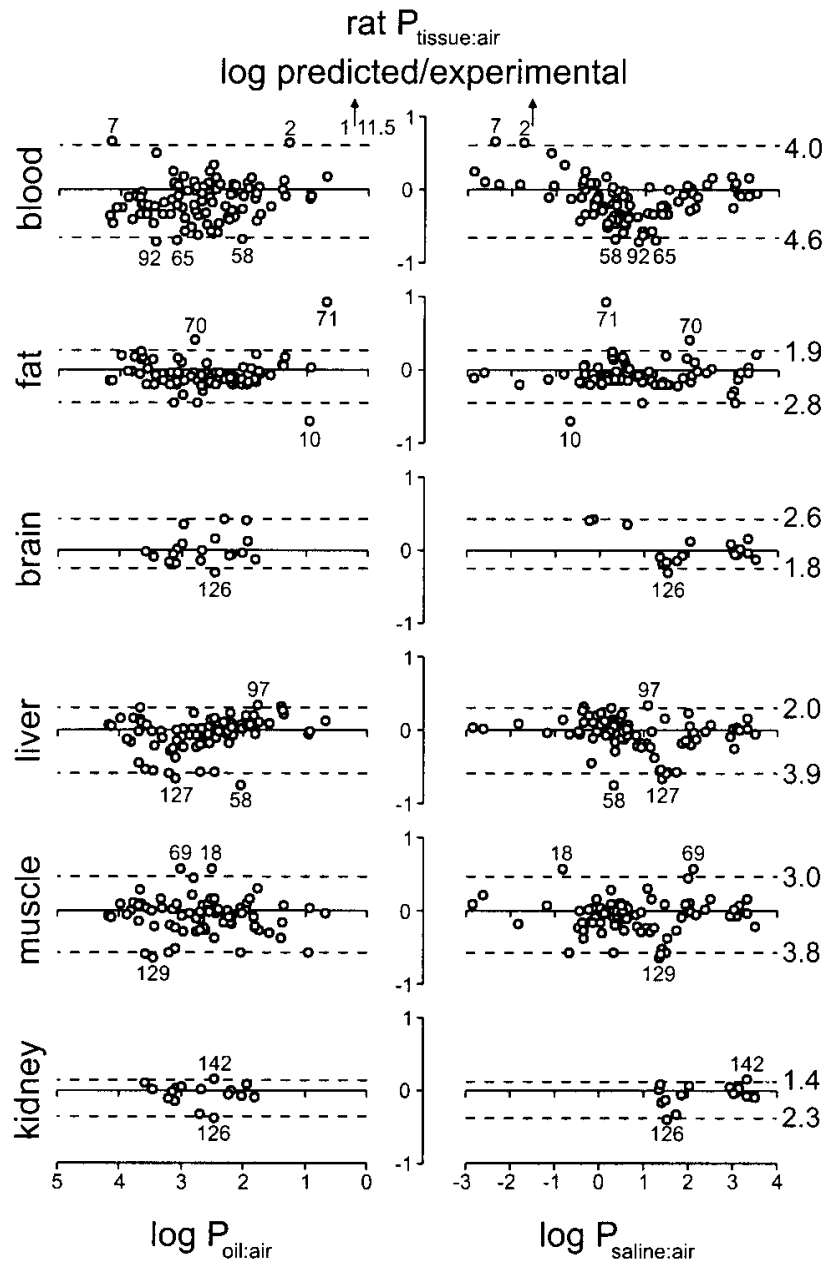

FIG. 5. Double-log representation of the ratio of predicted and experimentally determined $P_{\text {tissue:air }}$ against $P_{\text {oil:air }}$ and against $P_{\text {saline:air }}$ for rat blood, fat, brain, liver, muscle, and kidney as indicated. Results are presented in two dimensions for a clear insight into the distribution of all points relative to the fitted regression planes. The 2.5 and 97.5 percentiles are drawn (dashed lines) and the values of the percentiles are indicated at the right for each tissue. Outliers are identified by their compound numbers. $n$-Ethane (compound 1 ), which lies beyond the borders of the graph, has been indicated by arrows together with the value of the ratio (11.5).

a proportional relation between tissue:saline and oil:air partition coefficients is not clear. Fitting power functions to the nontransformed data of Table 1 by nonlinear regression, as an alternative for logarithmic transformation, did not result in a significant improvement of the fits, generally yielded exponent values close to 1 , and caused a marked increase in crosscorrelation between the fitted parameters, indicating that the addition of the exponents in the equation caused redundancy in the parameters.

Inclusion of the constant term $c$ in Eq. (1) did not improve the linear regressions and did not cause significant changes in $\alpha_{\mathrm{o}}$ and $\alpha_{\mathrm{s}}$. With the exception of fat tissue, the magnitude of the fitted constant was consistently small and its $\mathrm{CV}$ value was consistently large (up to over 1000\%). The constant $c$ in Eq. (1) appeared to be redundant to a certain extent, because it caused one order of magnitude increase in the cross-correlation coefficients of the fitted parameters and failed to improve the correlation coefficients of the fits. However, neglecting the constant resulted in the underestimation of the mean tissue partition coefficients by up to $20 \%$. Although the physical meaning of the constant is unclear, it may compensate for small systematic deviations in tissue partitioning or for small systematic errors in the values of reported partition coefficients. The relatively large values estimated for the intercepts for human and rat fat (Tables 2 and 3) cannot be explained at present. It should be noted, however, that the fat partition coefficients of compounds included in the regressions are generally large.

For the majority of compounds $(>80 \%) P_{\text {saline:air }}$ values were collected from the literature. For the remaining compounds either $P_{\text {water:air }}$ values were reported $(\sim 10 \%)$ or it is unclear whether the published values represent $P_{\text {water:air }}$ or $P_{\text {saline:air }}$ $(\sim 10 \%)$. For volatile anesthetics, it has been shown that $P_{\text {saline:air }}=0.87-0.97 P_{\text {water:air }}$ (Steward et al., 1973; Renzi and Waud, 1977; Halliday et al., 1977; Lerman et al., 1983). The magnitude of the difference is similar to that of the experimental error in the determination of the partition coefficient (e.g., see Sato and Nakajima, 1979a,b). The small difference between $P_{\text {water:air }}$ and $P_{\text {saline:air }}$ and the minority of compounds to which this applies indicates that the error introduced by ignoring the difference will not affect the results significantly.

In an alternative approach, VOC tissue partitioning is supposed to involve partitioning into specific tissue components, i.e., water, phospholipids, and neutral lipids (Poulin and Krishnan, 1995a,b, 1996a,b). The solubility of compounds in the various tissue fractions, estimated from partition coefficients in $n$-octanol or vegetable oil and in water, is used to calculate tissue partitioning. Reversible interactions with proteins or hemoglobin included in a study on blood partitioning are supposed to be due to the presence of hydrophobic binding pockets and the contribution of covalent interactions to VOC partitioning is considered negligible (Poulin and Krishnan, 1996b). The more detailed approach may contribute to unraveling processes and mechanisms involved in tissue partitioning. However, the more complex equations used (Poulin and Krishnan, 1995a) can be reduced to the simple form of Eq. (1). This shows that, for the prediction of VOC tissue partition coefficients in practice, prior knowledge of tissue composition is not required. Advantages of the simple approach are that it considers partitioning in tissues as a whole and that very few assumptions are required for the determination of regression coefficients from experimental data. A disadvantage is that the dimensionless regression coefficients $\alpha_{\mathrm{o}}$ and $\alpha_{\mathrm{s}}$, despite their relation to the relative proportions of hydrophilic and hydrophobic tissue constituents, are not associated with a specific process or mechanism.

The results (Tables 2 and 3 ) demonstrate that the regression of VOC blood partition coefficients is mainly determined by 
$\alpha_{\mathrm{s}}$, and the regression of VOC fat tissue partition coefficients is mainly determined by $\alpha_{\mathrm{o}}$. The marked differences in the regression coefficients for blood and fat are coherent with differences in tissue water and lipid contents. The regression of VOC partitioning in other tissues is intermediate but always with a major component determined by $\alpha_{\mathrm{s}}$. Differences in estimated regression coefficients for brain, liver, muscle, and kidney are less prominent than those for blood and fat. The values obtained for $\alpha_{\mathrm{s}}$ are remarkably constant within species and are an order of magnitude larger than values of $\alpha_{0}$, which are more variable but also less accurate as indicated by their larger CV values. It is concluded that, within species, the partitioning of the less lipophilic VOCs into brain, liver, muscle, and kidney is little tissue-dependent. Partitioning of lipophilic VOCs in these tissues will be moderately tissuedependent within species, as indicated by 3- to 10-fold differences in estimated values of $\alpha_{\mathrm{o}}$ for human and rat tissues, respectively.

A comparison between the two species shows a large, consistent difference in $\alpha_{\mathrm{s}}$ values, which range between 0.380 and 0.400 for human brain, liver, muscle, and kidney and between 0.772 and 0.878 for the corresponding rat tissues. Literature sources did not always reveal whether fresh or frozen tissues were used for the determination of partition coefficients. Since fresh and frozen tissues were used for both species, and since systematic differences in $\alpha_{\mathrm{o}}$ values are not observed between species, it seems highly unlikely that the difference in $\alpha_{\mathrm{s}}$ is caused by differences in the processing of rat and human tissues. This indicates that the factor 2 larger $\alpha_{\mathrm{s}}$ values for rat appear to reflect a genuine difference in rat and human tissue partitioning. Consequently, caution should be exercised in exchanging tissue partition coefficients between PBPK models for different species.

It has been reported before that partition coefficients for rat blood are higher than those for human blood (Gargas et al., 1989; Lam et al., 1990; Kaneko et al., 1994). When the bilinear model (Eq. (1)) is applied to the data of Gargas et al. (1989), who measured VOC partitioning in rat and human blood for 35 compounds in parallel, the species difference between the experimental values is reproduced. For 29 additional compounds the differences in rat and human blood partition coefficients, collected from various literature sources, are less pronounced and amount on average to a factor of 1.3 compared to 1.7 for the data of Gargas et al. (1989). A notable difference is that the compounds investigated by Gargas et al. (1989) are less hydrophilic than the additional 29 compounds. Apart from the data mentioned already in this section, we used data on 45 additional compounds for estimating the regression coefficients for human blood partitioning and on 28 additional, nonoverlapping compounds for estimating the regression coefficients for rat blood partitioning. In the final results of our bilinear regressions the species difference reported for the more restricted and more homogeneous set of data of Gargas et al. (1989) is no longer apparent. The variation in the very large dataset used for the regressions in the present study may obscure more subtle species differences in tissue partition coefficients. However, this does not detract from the point that the present approach shows that it is possible to obtain a fairly accurate prediction of tissue concentrations of VOCs without prior knowledge of particular properties of the chemical class and without prior knowledge on tissue composition.

The reliability of the predictions, as assessed from the ratio of predicted and experimental values, indicates that it is possible to predict the concentration of VOCs in human tissues with an accuracy of a factor of 4.0 and in rat tissues with an accuracy of a factor of 5.0. Thus, the concentrations of $95 \%$ of the VOCs considered in this study are predicted with an accuracy that appears to be sufficiently high to be used in human risk assessment. Reliable prediction of tissue partition coefficients will enable systematic PBPK modeling of exposurerelated brain concentrations of VOCs, which is essential to obtain insight into the relation between brain concentrations and adverse neurotoxic effects.

\section{ACK NOW LE D G MENTS}

We thank Drs. Joost de Jongh, Joop Hermens, and Wouter Vaes for useful discussions and comments on the manuscript. This study was financially supported by the Dutch Platform for Alternatives to Animal Testing (PAD) Grant 95-03.

\section{REFERENCES}

Andersen, M. E. (1991). Physiological modeling of organic compounds. Ann. Occup. Hyg. 35, 309-321.

Chortkoff, B. S., Laster, M. J., Koblin, D. D., Taheri, S., Eger, E. I., II, and Halsey, M. J. (1994). Pharmacokinetics do not explain the absence of an anesthetic effect of perfluoropropane or perfluoropentane. Anesth. Analg. 79, 234-237.

Coburn, C. M., and Eger, E. I., II (1986). The partial pressure of isoflurane or halothane does not affect their solubility in rabbit blood or brain or human brain: Inhaled anesthetics obey Henry's law. Anesth. Analg. 65, 960-962.

Droz, P. O. (1978). Contribution à la recherche d'indices biologiques d'exposition aux solvants. Détermination de leurs coefficients de partage et étude de leur comportement dans l'organisme à l'aide de modéles de simulation. Thesis. Neuchâtel University, Switzerland.

Eger, R. R., and Eger, E. I., II (1985). Effect of temperature and age on solubility of enflurane, halothane, isoflurane, and methoxyflurane in human blood. Anesth. Analg. 64, 640-642.

Eger, E. I., II (1987). Partition coefficients of I-653 in human blood, saline, and olive oil. Anesth. Analg. 66, 971-973.

Eger, E. I., II, Liu, J., Koblin, D. D., Laster, M. J., Taheri, S., Halsey, M. J., Ionescu, P., Chortkoff, B. S., and Hudlicky, T. (1994). Molecular properties of the "ideal" inhaled anesthetic: Studies of fluorinated methanes, ethanes, propanes, and butanes. Anesth. Analg. 79, 245-251.

Falk, A., Gullstrand, E., Lof, A., and Wigaeus-Hjelm, E. (1990). Liquid/air partition coefficients of four terpenes. Br. J. Ind. Med. 47, 62-64.

Fang, Z., Ionescu, P., Chortkoff, B. S., Kandel, L., Sonner, J., Laster, M. J., and Eger, E. I., II (1997a). Anesthetic potencies of $n$-alkanols: Results of additivity and solubility studies suggests a mechanism of action similar to that for conventional inhaled anesthetics. Anesth. Analg. 84, 1042-1048.

Fang, Z., Laster, M. J., Gong, D., Ionescu, P., Koblin, D. D., Sonner, J., Eger, 
E. I., II, and Halsey, M. J. (1997b). Convulsant activity of non-anesthetic gas combinations. Anesth. Analg. 84, 634-640.

Fang, Z., Sonner, J., Laster, M. J., Ionescu, P., Kandel, L., Koblin, D. D., Eger, E. I., II, and Halsey, M. J. (1996). Anesthetic and convulsant properties of aromatic compounds and cycloalkanes: Implications for mechanisms of narcosis. Anesth. Analg. 83, 1097-1104.

Fassoulaki, A., and Eger, E. I., II (1986). Starvation increases the solubility of volatile anaesthetics in rat liver. Br. J. Anaesth. 58, 327-329.

Fiserova-Bergerova, V., and Diaz, M. L. (1986). Determination and prediction of tissue-gas partition coefficients. Int. Arch. Occup. Environ. Health 58, $75-87$.

Fiserova-Bergerova, V., Tichy, M., and Di Carlo, F. J. (1984). Effects of biosolubility on pulmonary uptake and disposition of gases and vapors of lipophilic chemicals. Drug Metab. Rev. 15, 1033-1070.

Gargas, M. L., Burgess, R. J., Voisard, D. E., Cason, G. H., and Andersen, M. E. (1989). Partition coefficients of low-molecular weight volatile chemicals in various liquids and tissues. Toxicol. Appl. Pharmacol. 98, 87-99.

Gargas, M. L., Medinsky, M. A., and Andersen, M. E. (1995). Pharmacokinetic modeling approaches for describing the uptake, systemic distribution, and disposition of inhaled chemicals. Crit. Rev. Toxicol. 25, 237-254.

Halliday, M. M., MacDonald, I., and MacGregor, M. H. G. (1977). Gas chromatographic determination of Ostwald solubility coefficients for cyclopropane, halothane and trichlorethene (trichloroethylene). Br. J. Anaesth. 49, 413-417.

Järnberg, J., and Johanson, G. (1995). Liquid/air partition coefficients of the trimethyl-benzenes. Toxicol. Ind. Health 11, 81-88.

Johanson, G., and Dynésius, B. (1988). Liquid/air partition coefficients of six commonly used glycol ethers. Br. J. Ind. Med. 45, 561-564.

Kaneko, T., Wang, P. Y., and Sato, A. (1994). Partition coefficients of some acetate esters and alcohols in water, blood, olive oil and tissues. Occup. Environ. Med. 51, 68-72.

Krishnan, K., and Andersen, M. E. (1994). Physiologically based pharmacokinetic modeling in toxicology. In Principles and Methods of Toxicology. 3rd ed. (A. W. Hayes, Ed.), pp. 149-188. Raven Press, New York.

Lam, C. W., Galen, T. J., Boyd, J. F., and Pierson, D. L. (1990). Mechanism of transport and distribution of organic solvents in blood. Toxicol. Appl. Pharmacol. 104, 117-129.

Lerman, J., Gregory, G. A., and Eger, E. I., II (1987). Effects of anaesthesia and surgery on the solubility of volatile anaesthetics in blood. Can. J. Anaesth. 34, 14-16.

Lerman, J., Schmitt-Bantel, B. I., Gregory, G. A., Willis, M. M., and Eger, E. I., II (1986). Effect of age on the solubility of volatile anesthetics in human tissues. Anesthesiology 65, 307-311.

Lerman, J., Willis, M. M., Gregory, G. A., and Eger, E. I., II (1983). Osmolarity determines the solubility of anesthetics in aqueous solutions at $37^{\circ} \mathrm{C}$. Anesthesiology 59, 554-558.

Liu, J., Laster, M. J., Taheri, S., Eger, E. I., II, Chortkoff, B. S., and Halsey, M. J. (1994). Effect of $n$-alkane kinetics in rats on potency estimation and the Meyer-Overton hypothesis. Anesth. Analg. 79, 1049-1055.

Mikkelsen, S. (1997). Epidemiological update on solvent neurotoxicity. Environ. Res. 73, 101-112.
Nihlén, L. A., and Johanson, G. (1995). Liquid/air partition coefficients of methyl and ethyl $t$-butyl ethers, $t$-amyl methyl ether, and $t$-butyl alcohol. $J$. Expo. Anal. Environ. Epidemiol. 5, 573-582.

Paterson, S., and Mackay, D. (1989). Correlation of tissue, blood and air partition coefficients of volatile organic chemicals. Br. J. Ind. Med. 46, 321-328.

Perbellini, L., Brugnone, F., Caretta, D., and Maranelli, G. (1985). Partition coefficients of some industrial aliphatic hydrocarbons (C5-C7) in blood and human tissues. Br. J. Ind. Med. 42, 162-167.

Pierce, C. H., Dills, R. L., Silvey, G. W., and Kalman, D. A. (1996). Partition coefficients between human blood or adipose tissue and air for aromatic solvents. Scand. J. Work Environ. Health 22, 112-118.

Poulin, P., and Krishnan, K. (1995a). A biologically-based algorithm for predicting human tissue:blood partition coefficients of organic chemicals. Hum. Exp. Toxicol. 14, 273-280.

Poulin, P., and Krishnan, K. (1995b). An algorithm for predicting tissue:blood partition coefficients of organic chemicals from $n$-octanol:water partition coefficient data. J. Toxicol. Environ. Health 46, 101-113.

Poulin, P., and Krishnan, K. (1996a). A tissue composition-based algorithm for predicting tissue:air partition coefficients or organic chemicals. Toxicol. Appl. Pharmacol. 136, 126-130.

Poulin, P., and Krishnan, K. (1996b). A mechanistic algorithm for predicting blood:air partition coefficients of organic chemicals with the consideration of reversible binding in hemoglobin. Toxicol. Appl. Pharmacol. 136, 131137.

Renzi, F., and Waud, B. E. (1977). Partition coefficients of volatile anesthetics in Krebs' solution. Anesthesiology 47, 62-63.

Sato, A., and Nakajima, T. (1979a). Partition coefficients of some aromatic hydrocarbons and ketones in water, blood and oil. Br. J. Ind. Med. 36, 231-234.

Sato, A., and Nakajima, T. (1979b). A structure-activity relationship of some chlorinated hydrocarbons. Arch. Environ. Health 34, 69-75.

Steward, A., Allott, P. R., Cowles, A. L., and Mapelson, W. W. (1973). Solubility coefficients for inhaled anaesthetics for water, oil and biological media. Br. J. Anaesth. 45, 282-293.

Strum, D. P., and Eger, E. I., II (1987). Partition coefficients for sevoflurane in human blood, saline, and olive oil. Anesth. Analg. 66, 654-656.

Taheri, S., Laster, M. J., Liu, J., Eger, E. I., II, Halsey, M. J., and Koblin, D. D. (1993). Anesthesia by $n$-alkanes not consistent with the Meyer-Overton hypothesis: Determinations of the solubilities of alkanes in saline and various lipids. Anesth. Analg. 77, 7-11.

Tardif, R., Charest-Tardif, G., Brodeur, J., and Krishnan, K. (1997). Physiologically based pharmacokinetic modeling of a ternary mixture of alkylbenzenes in rats and humans. Toxicol. Appl. Pharmacol. 144, 120-134.

Yasuda, N., Targ, A. G., and Eger, E. I., II (1989). Solubility of I-563, sevoflurane, isoflurane, and halothane in human tissues. Anesth. Analg. 69, 370-373.

White, R. F., and Proctor, S. P. (1997). Solvents and neurotoxicity. Lancet 349, 1239-1243. 